\title{
Étude du rayonnement UV d'une lampe à décharge mercure haute pression en fonction de son mode d'alimentation
}

\author{
T. Pousset, D. Porras, H. Elloumi et G. Zissis
}

Centre de Physique des Plasmas et de leurs Applications de Toulouse, 118 route de Narbonne, 31062 Toulouse cedex, France

Résumé : Le travail présenté concerne l'alimentation et l'étude expérimentale du flux spectrique énergétique d'une lampe à décharge mercure haute pression spécialement destinée à la production de rayonnement UV. Ce type de source est tout particulièrement utilisé pour certains traitements de surface.

\section{MONTAGE EXPERIMENTAL}

Les lampes à décharge haute pression sont de plus en plus utilisées tant pour l'éclairage que pour les applications industrielles. La maîtrise de la répartition spectrale énergétique du flux rayonné conditionne l'efficacité et la flexibilité du système aussi bien que la qualité finale du produit. Si la constitution de la lampe joue un rôle primordial dans la distribution spectrique du rayonnement, celui des conditions d'alimentation est loin d'être négligeable.

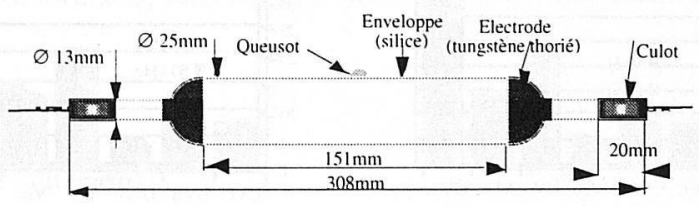

Figure 1 : La lampe étudiée

La lampe étudiée a été choisie comme représentative d'une famille de lampe allant de 1,3 à $16 \mathrm{~kW}$. Ces caractéristiques sont $P_{\text {arc }}=1300 \mathrm{~W}, I_{\mathrm{arc}}=5,7 \mathrm{~A}_{\mathrm{eff}}, \mathrm{V}_{\mathrm{arc}}=245 \mathrm{~V}_{\mathrm{eff}}$, $\mathrm{V}_{\text {amorçage }}=900 \mathrm{~V}_{\text {crête }}, \mathrm{P}_{\mathrm{Hg}}=1,7 \mathrm{~atm}$, masse $\mathrm{Hg}_{\mathrm{Hg}}=80 \mathrm{mg}$, gaz tampon $=$ Argon (8 torr)

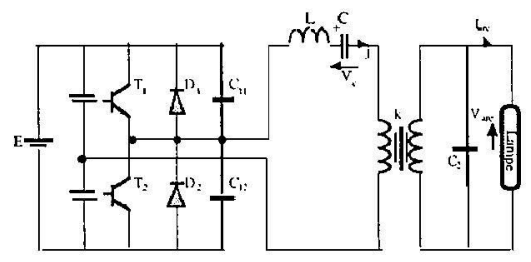

Figure 2 : Convertisseur à résonance série fonctionnant en mode thyristor dual 
Actuellement l'utilisation des convertisseurs à résonance dans la conversion de l'énergie électrique est aujourd'hui en plein essor. La commutation douce des interrupteurs limite les pertes par commutation dans les semi-conducteurs et autorise donc des fonctionnements à des fréquences élevées. L'encombrement des composants magnétiques (transformateur, inductances) est ainsi considérablement réduit. Pour toutes ces raisons nous envisageons d'alimenter notre lampe avec un onduleur à résonance.

Nous avons conçu, dimensionné et réalisé dans le cadre d'un projet européen commun (Projet Avicenna 54) un onduleur de tension à résonance fonctionnant à $50 \mathrm{kHz}$. Nous obtenons pour des lampes UV $1300 \mathrm{~W}$ et UV5000W les dimensions de ballast du tableau 1. L'onduleur à résonance réduit considérablement les dimensions du ballast de la lampe. L'amélioration est d'autant meilleure que la puissance est grande.

Tableau 1 : Dimensions des deux ballast ( $50 \mathrm{~Hz}$ et $50 \mathrm{kHz}$ )

\begin{tabular}{|c|c|c|}
\cline { 2 - 3 } \multicolumn{1}{c|}{} & Ballast inductif $50 \mathrm{~Hz}$ & Onduleur de tension $50 \mathrm{kHz}$ \\
\hline \multirow{2}{*}{ Poids } & $1300 \mathrm{~W} \rightarrow>21 \mathrm{~kg}$ & $1300 \mathrm{~W} \rightarrow 8 \mathrm{~kg}$ \\
& $5000 \mathrm{~W} \rightarrow>60 \mathrm{~kg}$ & $5000 \mathrm{~W} \rightarrow>12 \mathrm{~kg}$ \\
\hline \multirow{2}{*}{ Volume } & $1300 \mathrm{~W} \rightarrow>14$ litres & $1300 \mathrm{~W} \rightarrow>6$ litres \\
\hline
\end{tabular}

\section{FLUX SPECTRIQUE ET RENDEMENT ENERGETIQUE}

Nous avons étudié la puissance rayonnée de la lampe alimentée avec ce convertisseur et comparé les résultats avec donnés par l'alimentation industrielle $50 \mathrm{~Hz}$.

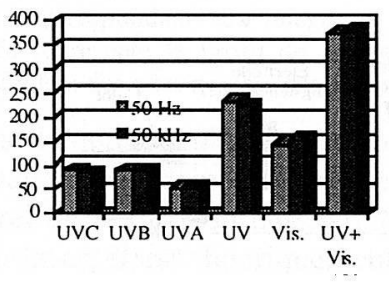

a)

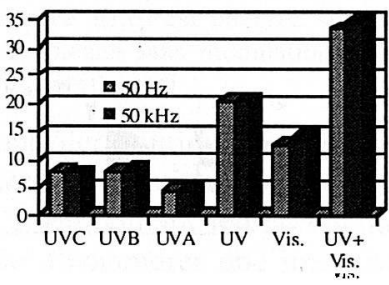

b)

Figure 3 : Comparaison entre le $50 \mathrm{~Hz}$ et le $50 \mathrm{kHz}$ - a) Flux (W) - b) Rendement énergétique (\%)

Du strict point de vue du rendement énergétique, il semble qu'il n'y ait pas d'intérêt d'alimenter la lampe avec un onduleur. Cependant ce type d'alimentation offre une grande marge de réglage de la puissance donc du flux spectrique comme le montre la figure 4.
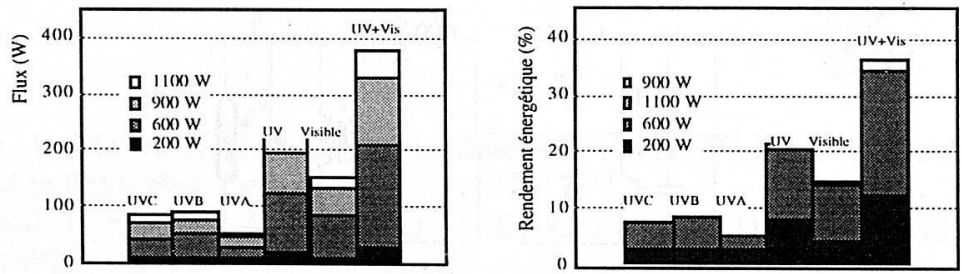

Figure 4 : Flux et rendement énergétique pour différentes puissances 